\title{
The Small Protein CP12: A Protein Linker for Supramolecular Complex Assembly
}

\author{
Emmanuelle Graciet, ${ }^{\ddagger}$ Pierre Gans, ${ }^{\S}$ Norbert Wedel," Sandrine Lebreton, ${ }^{\ddagger}$ Jean-Michel Camadro, ${ }^{\ddagger}$ and \\ Brigitte Gontero*, \\ Laboratoire d'ingéniérie des protéines et contrôle métabolique, Département Biologie des génomes, Institut Jacques Monod, \\ UMR 7592 CNRS, Universités Paris VI -VII, 2 place Jussieu, 75251 Paris Cedex 05, France, Institut de Biologie Structurale \\ "Jean-Pierre Ebel”, CEA-CNRS, 41 Avenue Jules Horowitz, 38027 Grenoble Cedex, France, and Botanisches Institut der \\ Christian-Albrechts-Universität zu Kiel, Am Botanischen Garten 1-9, 24118 Kiel, Germany
}

Received March 24, 2003; Revised Manuscript Received May 16, 2003

\begin{abstract}
CP12 is an 8.5-kDa nuclear-encoded chloroplast protein, isolated from higher plants. It forms part of a core complex of two dimers of phosphoribulokinase (PRK), two tetramers of glyceraldehyde 3-phosphate dehydrogenase (GAPDH), and CP12. The role of CP12 in this complex assembly has not been determined. To address this question, we cloned a cDNA encoding the mature CP12 from the green alga Chlamydomonas reinhardtii and expressed it in Escherichia coli. Sequence alignments show that it is very similar to other $\mathrm{CP} 12 \mathrm{~s}$, with four conserved cysteine residues forming two disulfide bridges in the oxidized CP12. On the basis of reconstitution assays and surface plasmon resonance binding studies, we show that oxidized, but not reduced, $\mathrm{CP} 12$ acts as a linker in the assembly of the complex, and we propose a model in which CP12 associates with GAPDH, causing its conformation to change. This GAPDH/ CP12 complex binds PRK to form a half-complex (one unit). This unit probably dimerizes due partially to interactions between the enzymes of each unit. Reduced CP12 being unable to reconstitute the complex, we studied the structures of oxidized and reduced CP12 by NMR and circular dichroism to determine whether reduction induced structural transitions. Oxidized CP12 is mainly composed of $\alpha$ helix and coil segments, and is extremely flexible, while reduced CP12 is mainly unstructured. Remarkably, CP12 has similar physicochemical properties to those of "intrinsically unstructured proteins" that are also involved in regulating macromolecular complexes, or in their assembly. CP12s are thus one of the few protein families of intrinsically unstructured proteins specific to plants.
\end{abstract}

In recent years, the use of very sensitive analytical techniques has revealed the presence of small proteins in macromolecular complexes. Their presence raises questions about their role in the formation and regulation of higher order structures $(1-3)$.

CP12 is a small, $8.5-\mathrm{kDa}$ nuclear-encoded chloroplast protein that has been isolated and cloned from a few eukaryotic and prokaryotic photosynthetic organisms (46 ). Many expressed sequence tags (ESTs) encoding putative CP12 proteins have been found in a wide variety of photosynthetic organisms in the past few years $(7,8)$. CP12 has been isolated as part of a core complex composed of CP12, phosphoribulokinase (PRK), and glyceraldehyde 3-phosphate dehydrogenase (GAPDH). ${ }^{1}$ These enzymes are regulated by light and are involved in $\mathrm{CO}_{2}$ assimilation in photosynthetic organisms (Benson-Calvin cycle). Interestingly, CP12 shares sequence homologies with the C-terminal extension of GapB, a GAPDH subunit in the chloroplasts of higher plants (4). This C-terminal end is responsible for the

* To whom correspondence should be addressed. Tel: 3314427 47 41. Fax: 331442757 16. E-mail: meunier@ijm.jussieu.fr.

$\doteqdot$ CNRS Universités Paris VI -VII

$\S$ CEA-CNRS.

"Botanisches Institut der Christian-Albrechts-Universität zu Kiel.

${ }^{1}$ Abbreviations: GAPDH: glyceraldehyde 3-phosphate dehydrogenase, PRK: phosphoribulokinase, CD: circular dichroism, NMR: nuclear magnetic resonance, SPR: surface plasmon resonance; IUP: intrinsically unstructured protein. oligomerization of the higher plant GAPDH $\left(\mathrm{A}_{2} \mathrm{~B}_{2}\right)$ into a hexadecamer $\left(\mathrm{A}_{8} \mathrm{~B}_{8}\right)$, which is regulated by metabolites such as 1,3-biphosphoglyceric acid $(9,10)$. In green algae, GAPDH is present as an $\mathrm{A}_{4}$ homotetramer which has not been shown to oligomerize to form a regulatory GAPDH.

Our group isolated a PRK/GAPDH complex, composed of two dimers of PRK and two homotetramers $\mathrm{A}_{4}$ of GAPDH, from the green alga Chlamydomonas reinhardtii (11). Recent cryo-electron microscopy studies suggested that other components are part of this complex (12). As Wedel et al (5) have shown that CP12 occurs with GAPDH and PRK in $C$. reinhardtii, this small protein was a good candidate. Recent mass spectrometry and kinetic studies on native GAPDH (isolated from the green alga) showed that it was a complex of GAPDH and CP12 (13), confirming that the complex described earlier by Avilan et al (11) was the same as the one isolated by Wedel et al (5). The activities of the enzymes involved in this complex are regulated in vitro by metabolites such as $\operatorname{NADP}(\mathrm{H})(5,6,14)$ and thioredoxins or the nonphysiological reducing agent DTT (15).

A partial sequence of CP12 in C. reinhardtii was obtained by PCR (5) (accession number in TrEMBL: 065775), but the $5^{\prime}$ and $3^{\prime}$ ends corresponding to the $\mathrm{N}$ - and C-terminal parts of the mature protein were missing. We have therefore cloned the entire cDNA of algal CP12 and developed an expression system in Escherichia coli. 
Previous studies on recombinant GAPDH (devoid of CP12) and native GAPDH (associated with CP12) demonstrated that the association of CP12 with GAPDH was responsible for changes in the kinetic parameters of GAPDH, probably due to conformation changes. These results already defined a new function for CP12, different from the regulatory one described earlier $(5,6)$. Nevertheless, since the highly homologous C-terminal extension of gapB is involved in the oligomerization of higher plant GAPDH, and given that CP12 is also part of the PRK/CP12/GAPDH complex, it may also play a role in the assembly of the complex. We have therefore studied its role under oxidized and reduced state in the assembly of the PRK/CP12/GAPDH complex from $C$. reinhardtii. We used surface plasmon resonance binding experiments to determine how this complex might be assembled and for the first time, we propose a model for its assembly pathway.

Since oxidized and reduced CP12 do not have the same behavior regarding complex formation, and since no structural data were available for any of the cloned CP12 proteins, we analyzed the structure of oxidized and reduced CP12 in solution by NMR spectroscopy and circular dichroism (CD). We also linked these results with the role of CP12 in the assembly of the PRK/CP12/GAPDH complex.

\section{EXPERIMENTAL PROCEDURES}

cDNA Library Construction. C. reinhardtii (cw15) was grown photoautotrophically for 4 days under a 16/8 h light/ dark regime in high salt medium (16) with $5 \% \mathrm{CO}_{2}$ in the air during the light phase. The exponentially growing cells were then harvested 2.5 (A) and $6 \mathrm{~h}$ (B) after light setting. Nucleic acids were isolated by three rounds of phenol/ chloroform and a final chloroform extraction. The total nucleic acids were precipitated with potassium acetate/2propanol and separated by $\mathrm{LiCl}$ precipitation. The polyadenylated RNA was purified by chromatography on oligo-dT cellulose (Pharmacia, Freiburg) and cDNA was synthesized using a Pharmacia (Freiburg) kit. Double-stranded adapterlinked cDNAs were ligated into the dephosphorylated arms of $\lambda \mathrm{gt} 11$ and packed into Gigapack II Gold phage extracts (Stratagene, La Jolla). The resulting phages were incubated with E. coli strain Y1088 for $20 \mathrm{~min}$ at room temperature, plated on BBL-agar, and the plates incubated at $37{ }^{\circ} \mathrm{C}$ overnight. The resulting plaques were overlaid with SM to elute the phages. The elutes were centrifuged and the cleared phage-containing supernatants were adjusted to $7 \%$ DMSO. Aliquots of the two libraries (A and B) were stored at -80 ${ }^{\circ} \mathrm{C}$. The titers of the libraries were about $10^{8} \mathrm{pfu} / \mathrm{mL}$, containing about $90 \%$ recombinant phages.

Cloning of C. Reinhardtii CP12. Primers were designed from ESTs available at NCBI Genbank (AV626097 and AV634344) (17). They were used to amplify directly CP12 (forward primer: 5'CTAGCGGCCAGCCCGCTGTG3' and reverse primer: 5'GAATTAGGAGCATCTGCGCCG3') from the $C$. reinhardtii cDNA library mentioned above. The PCR product (248 bp) was cloned in pGEM-T (Promega) and sequenced. The primers against the partial sequence of $C$. reinhardtii published in ref 5 were also used (forward: 5'CCGGTTAGTGGAGAATGCG3' and reverse: 5'GGTTATCCTTACAGTAATCC3').

Overexpression and Purification of C. reinhardtii CP12. The N-terminal sequence of native CP12 was obtained from
C. reinhardtii purified PRK/CP12/GAPDH complex in solution (Edman method). The N-terminal sequences of PRK and GAPDH are known to be DKDKT and EKKIR, respectively. The remaining amino acid residues were thus attributed to CP12. Primers containing the NdeI and XhoI restriction sites were used to amplify and clone mature CP12 cDNA in frame with the N-terminal histidine tag of the pET16b expression vector (Novagen) (forward primer 5'CATATGAGCGGCCAGCCCG3' and reverse primer 5'GAGCTCAATTAGGAGCAT CTGC3'). A cleavage site by factor $\mathrm{Xa}$ is present between the histidine tag and mature CP12.

C. reinhardtii $\mathrm{CP} 12$ cDNA was expressed in freshly transformed E. coli BL21(DE3)pLysS. Bacteria were grown in 2YT medium with $100 \mu \mathrm{g} / \mathrm{mL}$ ampicillin and $34 \mu \mathrm{g} / \mathrm{mL}$ chloramphenicol at $37{ }^{\circ} \mathrm{C}$ until the $\mathrm{OD}_{600}$ was 0.5 to 0.6 . Cultures were cooled on ice and CP12 synthesis was induced with $1 \mathrm{mM}$ isopropyl- $\beta$, D-thiogalactopyranoside (IPTG). Protein synthesis took place at $30^{\circ} \mathrm{C}$ overnight.

Bacteria were collected by centrifugation and suspended in $\mathrm{Ni}-\mathrm{NTA}$ buffer $\left(50 \mathrm{mM} \mathrm{NaH} \mathrm{PO}_{4}, 300 \mathrm{mM} \mathrm{NaCl}\right.$, and $10 \mathrm{mM}$ imidazol, $\mathrm{pH}$ 8.0). Cells were broken by sonication and centrifuged at $27000 \mathrm{~g}$ for $20 \mathrm{~min}$. The supernatant contained recombinant CP12 bearing a histidine tag (HisCP12). Recombinant His-CP12 was purified by metal ion affinity chromatography on Ni-NTA agarose (Qiagen) (1.2 $\times 8 \mathrm{~cm})$. The column was equilibrated in Ni-NTA buffer. Contaminants were first eluted out with $100 \mathrm{mM}$ imidazol. Apparently homogeneous His-CP12 was eluted with $200 \mathrm{mM}$ imidazol and stored at $-20^{\circ} \mathrm{C}$.

The His-CP12 used for in vitro complex reconstitution was digested with factor Xa (Novagen) (1u for $33 \mu \mathrm{g}$ of CP12). The sample was applied to a gel filtration Sephadex G50 (1 $\times 50 \mathrm{~cm}$ ) column in $50 \mathrm{mM} \mathrm{NaH}_{2} \mathrm{PO}_{4}, 50 \mathrm{mM} \mathrm{NaCl}, \mathrm{pH}$ 7.5 to separate $\mathrm{CP} 12$ from factor $\mathrm{Xa}$ and the histidine tag. Purified CP12 was stored at $-20^{\circ} \mathrm{C}$.

The PRK/CP12/GAPDH complex was purified from $C$. reinhardtii $\mathrm{WM}^{-}$as in ref 11 . PRK and GAPDH may also be purified separately to obtain the native isolated enzymes (11). Recombinant GAPDH was purified as in ref 13 .

Redox State of CP12. The free SH groups in CP12 were quantified by alkylation of the reactive cysteine residues with iodoacetamide. Iodoacetamide was prepared as in ref 18 . Oxidized and reduced CP12 were alkylated with $100 \mathrm{mM}$ iodoacetamide for $30 \mathrm{~min}$ at room temperature and in the dark. Samples were desalted on a Zip-tip $\mathrm{C}_{18}$ and analyzed by mass spectrometry (MALDI-TOF). Alkylation of one cysteine residue increased the mass by $57.27 \mathrm{Da}$. Reduced CP12 was obtained by incubating the protein with $30 \mathrm{mM}$ DTT for $1 \mathrm{~h}$ at $30^{\circ} \mathrm{C}$.

In Vitro Complex Reconstitution. Recombinant GAPDH and native PRK were dialyzed separately against reconstitution buffer (30 mM Tris, $100 \mathrm{mM} \mathrm{NaCl}, 2$ mM EDTA, 0.1 mM NAD, pH 7.9) supplemented with $5 \mathrm{mM}$ Cys or $1 \mathrm{mM}$ DTT on a YM30 centricon (Amicon, Millipore). Oxidized or reduced CP12 was added in a molar ratio of 1 monomer of CP12 to 1 tetramer of GAPDH and 1 dimer of PRK (1: 1:1) to reconstitute the complex. The proteins were dialyzed again in the same buffer and concentrated to $50 \mu \mathrm{L}$ on a YM10 centricon (Amicon - Millipore). $10 \%$ glycerol was added and the proteins were incubated for $1 \mathrm{~h}$ at $30^{\circ} \mathrm{C}$ and kept at $4{ }^{\circ} \mathrm{C}$ overnight or longer. 
Surface Plasmon Resonance (SPR) Binding Experiments. Oxidized CP12 or His-CP12 (ligand) was immobilized on CM5 sensor chips in HBS-EP buffer (10 mM Hepes, 150 $\mathrm{mM} \mathrm{NaCl}, 3.4 \mathrm{mM}$ EDTA, 0.005\% (v/v) P20, pH 7.4; BIAcore) by amine coupling using the amine coupling kit supplied by BIAcore. The aim of $50 \mathrm{RU}$ (resonance unit) was reached. $785 \mathrm{RU}$ of reduced PRK were immobilized on a CM5 chip by SH coupling.

A range of protein (analyte) concentrations were dialyzed against buffer HBS-EP and $0.1 \mathrm{mM}$ NAD and used to determine the dissociation constants with a BIACORE 2000 instrument. In each interaction run, the analyte was injected for $3 \mathrm{~min}$ to record the association phase to the flow cells, after which buffer was injected for 3 min to record the dissociation phase. The association and dissociation phases were used to derive the dissociation and association rates ( $k_{\mathrm{d}}$ and $k_{\mathrm{a}}$, respectively). The equilibrium-dissociating constant $\left(K_{\mathrm{d}}\right)$ is given by $K_{\mathrm{d}}=k_{\mathrm{d}} / k_{\mathrm{a}}$. Global fittings of the exponential curves (sensorgrams) giving both $k_{\mathrm{a}}$ and $k_{\mathrm{d}}$ values and the response at equilibrium $\left(R_{\mathrm{eq}}\right)$ in RU were performed using the BIAeval 3.1 program using a model equivalent to the 1:1 Langmuir model for adsorption to a surface.

Circular Dichroism Studies on His-CP12. Spectra were recorded on a Jasco-810 dicrograph and analyzed with the programs Dicroprot (http://dicroprot-pbil.ibcp.fr/) and CDPro (http://lamar.colostate.edu/ sreeram/CDPro/main.html). All CD spectra are averages of four accumulations from 260 to $180 \mathrm{~nm}$ with a scan speed of $10 \mathrm{~nm} / \mathrm{min}$. His-CP12 was dialyzed against phosphate buffer $\left(10 \mathrm{mM} \mathrm{Na}_{2} \mathrm{HPO}_{4}-\mathrm{NaH}_{2}-\right.$ $\mathrm{PO}_{4}, \mathrm{pH}$ 6.0). Measurements were performed in $0.2 \mathrm{~cm}$ quartz cuvettes in a final volume of $300 \mu \mathrm{L}$.

NMR Studies of His-CP12. Homonuclear NMR experiments were recorded on His-tagged CP12 and CP12 in 50 $\mathrm{mM} \mathrm{NaH}_{2} \mathrm{PO}_{4}, 50 \mathrm{mM} \mathrm{NaCl}, \mathrm{pH} 6.0,10 \%$ (v/v) ${ }^{2} \mathrm{H}_{2} \mathrm{O}$. NMR heteronuclear studies were carried out on His-CP12 synthesized in M9 minimal medium containing $1 \mathrm{~g} / \mathrm{L}$ of $\left[{ }^{15} \mathrm{~N}\right]-$ ammonium chloride (Isotec) as the sole nitrogen source. The protein was purified on $\mathrm{Ni}-\mathrm{NTA}$ agarose. The samples were dialyzed against NMR buffer $\left(50 \mathrm{mM} \mathrm{NaH} \mathrm{PO}_{4}, 50 \mathrm{mM}\right.$ $\mathrm{NaCl}, \mathrm{pH}$ 6.0) and concentrated to $0.9 \mathrm{mM}$ in a final volume of $450 \mu \mathrm{L}$. A $50 \mu \mathrm{L}$ aliquot of ${ }^{2} \mathrm{H}_{2} \mathrm{O}(10 \% \mathrm{v} / \mathrm{v})$ was added and the sample was degassed with argon before recording.

2D homonuclear TOCSY spectra, NOESY spectra, $2 \mathrm{D}^{1} \mathrm{H}-$ ${ }^{15} \mathrm{~N}$ HSQC spectra (19), and heteronuclear nOe $\left\{{ }^{1} \mathrm{H}\right\}-{ }^{15} \mathrm{~N}$ spectra (20) were recorded at $27{ }^{\circ} \mathrm{C}$ on Varian Inova 400 and $600 \mathrm{MHz}$ spectrometers equipped with a triple-resonance $\left({ }^{1} \mathrm{H},{ }^{13} \mathrm{C},{ }^{15} \mathrm{~N}\right)$ probe, including shielded z-gradients. Spectra were processed using FELIX version 97.0 (Accelrys). Chemical shifts were measured with reference to the $\mathrm{H}_{2} \mathrm{O}$ signal taken at $27{ }^{\circ} \mathrm{C}$ relative to DSS. ${ }^{15} \mathrm{~N}$ chemical shifts used the $1 \mathrm{H} / \mathrm{X}$ frequency ratios as indirect references, as reported in ref 21 .

Mass Spectrometry Studies. MALDI-TOF mass spectra were obtained on a Voyager DE Pro mass spectrometer (Applied Biosystems). Samples were prepared as in ref 13 .

Miscellaneous Procedures. Antibodies raised against recombinant $\mathrm{CP} 12$ and GAPDH from $C$. reinhardtii were obtained after rabbits were given three injections of the purified proteins. Protein concentrations were determined with the Bio-Rad protein dye reagent, using bovine serum albumin as standard. Native gels were performed on a Phast System apparatus, with 4-15\% gradient gels (Pharmacia).
Proteins were transferred and revealed using GAPDH, CP12, or PRK antibodies.

\section{RESULTS}

C. Reinhardtii CP12 is Similar to Higher Plant CP12. PCR on the cDNA library constructed from $C$. reinhardtii $\mathrm{cw} 15$ strain gave a sequence identical to that of the EST AV634344. Genomic DNA from the WM3- ${ }^{-}$strain of $C$. reinhardtii could only be amplified with the primers corresponding to the sequence of the EST. N-terminal sequencing of CP12 within the PRK/CP12/GAPDH complex showed that the first amino acid residues of mature CP12 embedded in the complex were SGQPA. This sequence is identical to part of one translation frame of our PCR product. C. reinhardtii CP12 is highly homologous to other CP12s (Figure 1).

Recombinant CP12 is Mainly Oxidized and Contains two Disulfide Bridges. Mass spectrometry of purified recombinant CP12 with free thiol groups alkylated by iodoacetamide, showed a main peak at $\mathrm{m} / \mathrm{z} 8743$, corresponding to the unalkylated form of CP12. Given the small size of iodoacetamide, the absence of alkylation may be linked to the presence of two disulfide bridges. There were also two very weak peaks at $m / z 8800$ and 8856 , corresponding to partially reduced forms (data not shown).

$\mathrm{CP} 12$ was reduced with $30 \mathrm{mM}$ DTT at $30^{\circ} \mathrm{C}$ for $1 \mathrm{~h}$ and the four thiol groups were alkylated by iodoacetamide. Thus, all thiol groups were accessible after reduction (data not shown).

Role of CP12 as a Protein-Linker in the Assembly of the PRK/CP12/GAPDH Complex. CP12 has been isolated from a few eukaryotic and prokaryotic photosynthetic organisms as part of multi-enzyme complexes and the function of this small protein in complex assembly is unknown.

The complex can be reconstituted in vitro using native isolated PRK and GAPDH (purified from the green alga). This reconstituted complex had the same kinetic properties as the native one purified from $C$. reinhardtii (11). Yet, no complex could ever be reconstituted in vitro using isolated native PRK and recombinant GAPDH. The main difference was the replacement of native isolated GAPDH, which is in fact a complex of GAPDH and CP12, by recombinant GAPDH (devoid of CP12). Reconstitution assays were performed with native isolated PRK, recombinant GAPDH, and recombinant oxidized CP12. The addition of the small protein ensured the assembly of the complex (Figure 2). Thus, CP12 acts as a linker that is essential for complex assembly.

CP12 may also be obtained under a reduced state after treatment with a strong reducing agent such as DTT. We therefore checked whether reduced CP12 could still reconstitute the complex in vitro. Native PRK, recombinant GAPDH, and reduced CP12 were concentrated together and the complex reconstituted in the presence of $5 \mathrm{mM}$ Cys (Figure 2), but not in the presence of $1 \mathrm{mM}$ DTT. Two possibilities arose. The simplest was that reduced $\mathrm{CP} 12$ could be reoxidized prior to complex assembly when the mild reducing agent was used, but not in the presence of a stronger reducing agent such as DTT. The other possibility was that reduced CP12 could reconstitute the complex with formation of its disulfide bridges at the contact of either GAPDH or PRK in the presence of Cys. This would be prevented by 


\begin{tabular}{|c|c|c|}
\hline rabil & 1 & A TSEG - - . - E I SEKVEKSIQEAKETCA D D P V S GE CVA \\
\hline bi2 & 1 & 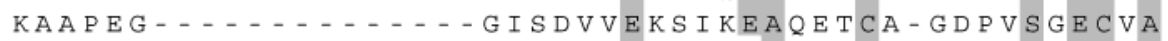 \\
\hline um & 1 & A A PE \\
\hline nacia & 1 & - A P DN $\cdots \cdots-\cdots$ R I SENVEKSIKEAQETCS - D D P V SGECVA \\
\hline Nicot & 1 & 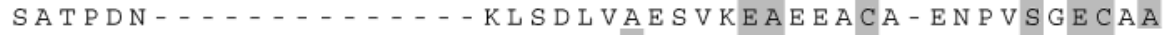 \\
\hline cabi3 & 1 & 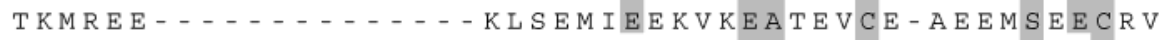 \\
\hline amy & 1 & 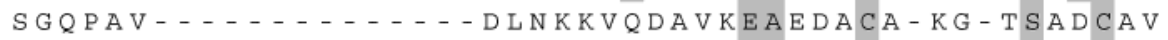 \\
\hline Nosto1 & 1 & MTTTLN-.-- SFETAGAANLEEAITEAITEARTTCELNGSDSANCAV \\
\hline ssto2 & 1 & MTMTFNVAPSGANSDNNGTTNLEKAILAAIAEARTTCEQNGDGSPNCAV \\
\hline Synec & 1 & 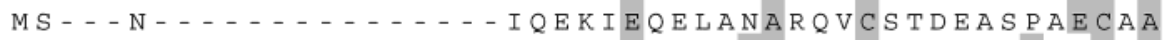 \\
\hline Thermo & 1 & 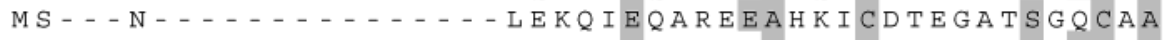 \\
\hline & 1 & 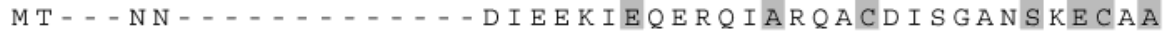 \\
\hline & 35 & AWDEVEELSAAASHARDKKKAGG- - SDPLEEYCNDNPETDECRTYDN \\
\hline Arabi2 & 35 & A W DEVEELSAAASHARDKKKADG- - SDPLEEYCKDNPETNECRTYDN \\
\hline Pist & 33 & AWDEVEELSAAASHARDRKKE- - S D PLEDYCKDNPETDECKTYDN \\
\hline acia & & AWDVVEELSAAASHARDKAKD- - VEPLEEYCKDNPETDECRTYDN \\
\hline Nic & 35 & A W DVVE EASAAASHARDKKKES- - S D P L ENY C K DN P E T DE C R T Y D N \\
\hline cabi3 & 35 & AWDEVEEVSQARADLRIKLKLLN- - QDPLESFCQENPETDECRIYED \\
\hline & 34 & A W D T VE L SAAVSHKKDAVKADVTLTDPLEA F K DAPDADECRVYED \\
\hline & 44 & A W D IVEELQAEKSDQQQ-AKAK- - KNSLENYCDRHPASVECLIYDV \\
\hline & 50 & A W D IVEELQAEKSHQQQ-AQKR--KS SLESFCDLHPEA LE C L IY DV \\
\hline & 32 & A W D AVEELEAEAAHQRQ - QHPT- - QT TLEKFCDENPDAAECR IYDD \\
\hline & 32 & A W DALEELQAEAAHQRAEQQDH- - K TSFQQY CDDNPDAAECR IY DD \\
\hline & 34 & EASHQKE- \\
\hline
\end{tabular}

FIGURE 1: Sequence of $C$. reinhardtii CP12 aligned with those of CP12s from other photosynthetic organisms and hypothetical sequences. Alignment was performed with ClustalW. Hypothetical sequences were obtained by BlastP with $C$. reinhardtii sequence as template. Arabi1, 2, and 3: Arabidopsis thaliana putative CP12s AAL32917, NP_191800, AAM62589, respectively; Pisum: Pisum sativum; Spina: Spinacia oleracea; Nicot: Nicotiana tabacum; Chlamy: Chlamydomonas reinhardtii; Nosto1 and 2: Nostoc punctiforme (ZP_00106092) and Nostoc sp. (NP_484948) (8), respectively; Synec: Synechocystis PCC6803; Thermo: Thermosynechococcus elongatus BP-1 (NP_682610) (7); Tricho: Trichodesmium erythraeum IMS101 (ZP_00071116). All accession numbers are from NCBI.

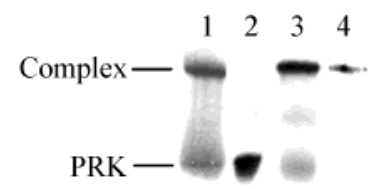

FIGURE 2: In vitro reconstitution assays with oxidized and reduced $\mathrm{CP} 12$. Proteins were transferred to a nitrocellulose membrane and revealed with antibodies raised against PRK. Lane 1: complex purified from $C$. reinhardtii (control; $2.4 \mu \mathrm{g}$ ). Lane 2 : in vitro reconstitution using native isolated PRK and recombinant GAPDH in a molar ratio of one dimer of PRK for one tetramer of GAPDH with $0.15 \mathrm{nmol}$ of each $(\approx 2 \mu \mathrm{g})$. Lane 3 : $\mathrm{CP} 12$ was added to the reconstitution mixture at a molar ratio of one monomer of CP12 for one dimer of PRK and one tetramer of GAPDH, using 0.15 nmol of each $(\approx 2 \mu \mathrm{g})$. Lane 4 : Complex reconstitution assay with $5 \mathrm{mM}$ Cys using reduced $\mathrm{CP} 12$, in the same molar ratio with 0.06 nmol of each $(0.5 \mu \mathrm{g})$.

DTT because of its higher reducing power. To test these possibilities, we incubated reduced CP12 with the reconstitution buffer supplemented with $5 \mathrm{mM}$ Cys or $1 \mathrm{mM}$ DTT overnight and alkylated CP12. $5 \mathrm{mM}$ Cys did not prevent the formation of the two disulfide bridges (no alkylation), whereas in the presence of $1 \mathrm{mM}$ DTT the four thiol groups were alkylated (data not shown).

Influence of CP12 on the Affinity of GAPDH for PRK. SPR studies with immobilized reduced PRK (ligand), and different concentrations of native GAPDH (GAPDH/CP12) (analyte) have shown that the dissociation constant $\left(K_{\mathrm{d}}\right)$ of the native GAPDH/PRK association is $62 \mathrm{nM}(22)$. We determined the dissociation constants of different subcomplexes by SPR to find how oxidized CP12 acts in complex assembly. The $K_{\mathrm{d}}$ was higher $(190 \mathrm{nM})$ when recombinant GAPDH (devoid of CP12) was used as analyte on immobilized PRK (ligand) than when native GAPDH was associated with PRK (Figure 3A).
The $K_{\mathrm{d}}$ was extremely low $(0.44 \mathrm{nM})$ when oxidized HisCP12 was used as ligand and recombinant GAPDH as analyte (Figure 3B). The curves were fitted to a pseudo 1:1 Langmuir model. The estimated $K_{\mathrm{d}}$ was much higher when PRK was used as analyte (1.3 $\mu \mathrm{M})$ (Figure 3C). Repetition of the experiments with oxidized CP12 showed that the tag did not affect the specificity of interaction.

Wedel et al proposed a model in which the formation of the complex involved the dimerization of two monomers of CP12 (6). We tested this hypothesis by measuring the $K_{\mathrm{d}}$ of oxidized CP12 on itself. The value obtained was high (51 $\mu \mathrm{M})$. The values obtained for the $K_{\mathrm{d}} \mathrm{S}$ are summarized in Table 1.

Structural Transitions of CP12 upon Reduction. To determine whether the specific role of oxidized CP12 in complex assembly, compared to reduced CP12, could be linked to structural transitions and not only to the presence of free thiol groups, we investigated the structure in solution of oxidized and reduced His-CP12 by CD and NMR.

CD spectra for oxidized CP12 showed two minima, at 222 and $205 \mathrm{~nm}$, indicating helical structures (Figure 4). Yet, the maximum at $192 \mathrm{~nm}$ was smaller than expected for an all $\alpha$-helix protein, probably because of the presence of random coils (minimum at $198 \mathrm{~nm}$ ). The estimation of protein secondary structure indicated that $\mathrm{CP} 12$ is composed of $\alpha$-helix with some random coil. Recombinant reduced CP12 was also analyzed by CD and NMR to determine the effect of reducing the two disulfide bridges on the structure of the protein. The CD spectra showed that CP12 underwent a dramatic conformational change after reduction and became even more disordered. The $\alpha$-helix content decreased, while those of random coils and undetermined structures increased (Figure 4). 

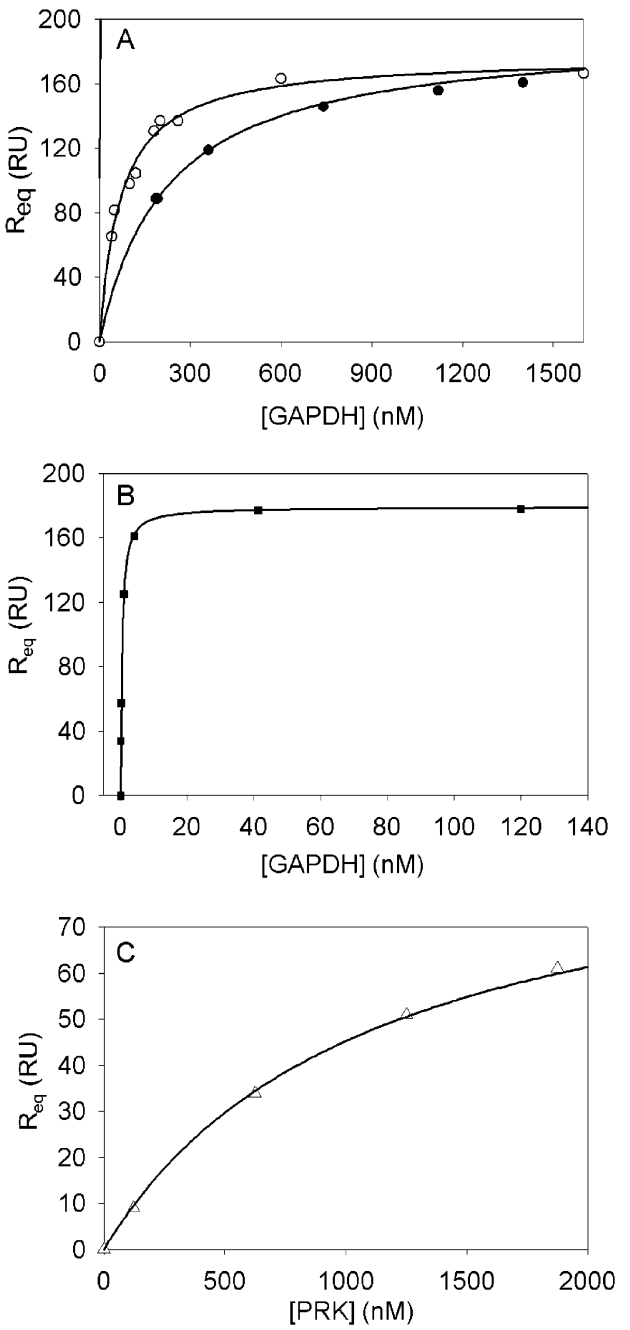

FIGURE 3: SPR binding experiments. The response at equilibrium $R_{\text {eq }}$ (in resonance unit (RU)) was reported as a function of the analyte concentration. The experimental points were fitted to a hyperbola: $R_{\mathrm{eq}}=\left(R_{\max }[\mathrm{GAPDH}]\right) /\left(K_{\mathrm{d}}+[\mathrm{GAPDH}]\right)$. Scatchard plots $\left(R_{\mathrm{eq}} /[\mathrm{GAPDH}]\right.$ versus $\left.R_{\mathrm{eq}}\right)$ were also used and the slope gave the same estimations (data not shown). (A) PRK was used as a ligand and native GAPDH $(O)$ or recombinant GAPDH $(\bullet)$ were used as analyte. (B and C) Oxidized His-CP12 was immobilized (ligand) and recombinant GAPDH (B) or native PRK (C) were used as analyte. The errors on the estimated parameters were less than $10 \%$ in all cases.

Table 1: Dissociation Constants Obtained by Surface Plasmon Resonance in Bold, the Lowest $K_{\mathrm{d}} \mathrm{s}$ Obtained

\begin{tabular}{lll}
\hline \multicolumn{1}{c}{ analyte } & $\mathrm{CP} 12_{\text {ox }}(\mathrm{M})$ & PRK $(\mathrm{M})$ \\
\hline CP12 $_{\text {ox }}$ & $0.51 \times 10^{-4}$ & nd \\
GAPDH $_{\text {rec. }}$ & $\mathbf{0 . 4 4} \times \mathbf{1 0}^{-9}$ & $0.19 \times 10^{-6}$ \\
PRK & $0.13 \times 10^{-5}$ & nd \\
GAPDH $_{\text {nat. }}$ & nd & $\mathbf{0 . 6 2} \times \mathbf{1 0}^{-\mathbf{7}}$ \\
\hline
\end{tabular}

To gain further insight into the structural transition of CP12 upon reduction and the mobility of reduced CP12, we also examined the effects of reduction by NMR. Figure 5 shows the HSQC spectrum of an oxidized sample of ${ }^{15} \mathrm{~N}-\mathrm{CP} 12$. Most of the resonances are localized in the range 8.5-8 ppm. The resonances of CP12 have not been assigned due to the superimposition of these signals and the absence of any nOe transfer in homonuclear NOESY experiments (data not shown). However, a first general conclusion on CP12 properties could be drawn from the spectra. Only about a

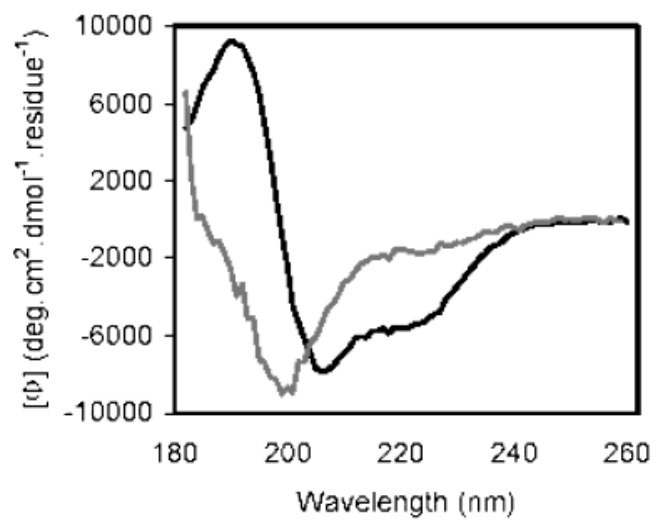

FIGURE 4: CD studies showing the structural changes of His-CP12 upon reduction. Spectra were obtained with $8 \mu \mathrm{M}$ of oxidized HisCP12 (black line) or reduced His-CP12 (grey line) in phosphate buffer $\mathrm{pH}$ 6.0.

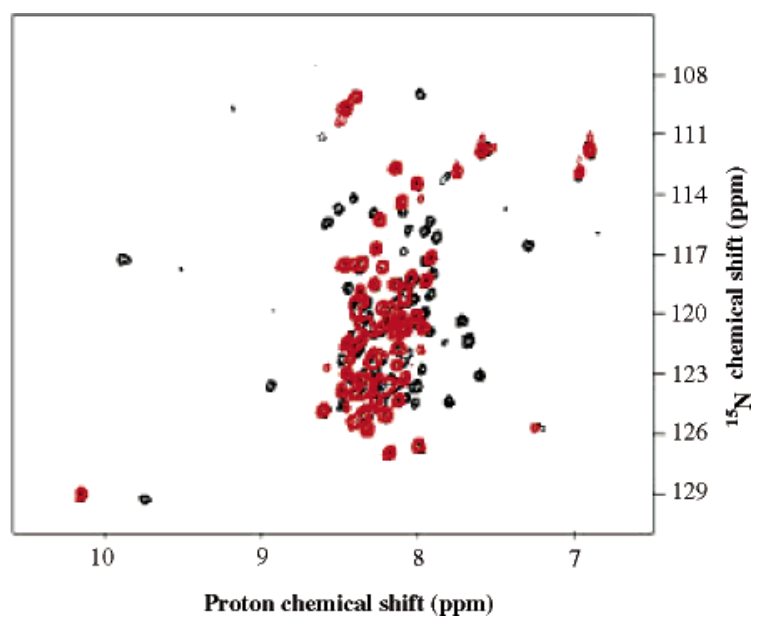

FIGURE 5: ${ }^{15} \mathrm{~N}-\mathrm{HSQC}$ spectra of the oxidized and reduced form of C. reinhardtii His-CP12. In black: $\mathrm{HSQC}$ spectrum at $400 \mathrm{MHz}$ of oxidized His-CP12 $(0.9 \mathrm{mM})$ at $27{ }^{\circ} \mathrm{C}$ in $50 \mathrm{mM} \mathrm{NaCl}, 50 \mathrm{mM}$ phosphate buffer, $\mathrm{pH}$ 6.0; four transients by $\mathrm{t} 1$ increment. In red: HSQC spectrum at $400 \mathrm{MHz}$ of reduced CP12 $(0.3 \mathrm{mM})$ at $27^{\circ} \mathrm{C}$ in $50 \mathrm{mM} \mathrm{NaCl}, 50 \mathrm{mM}$ phosphate buffer $\mathrm{pH} 6.0,10 \mathrm{mM}$ DTT; 16 transients by $\mathrm{t} 1$ increment.

third of the residues exhibit chemical shifts which are far from the random coil values (Figure 5), suggesting that CP12 is only partially structured. These features, together with the absence of nOe transfer strongly suggest that CP12 exhibits highly dynamic properties. This is further corroborated by ${ }^{1} \mathrm{H}-{ }^{15} \mathrm{~N}$ nOe experiments which show only very weak positive heteronuclear nOe effects indicative of high mobility (data not shown).

The reduction of oxidized $\mathrm{CP} 12$ caused a very large change in the NMR spectrum. All the correlations in the HSQC having chemical shift values different from those of a random coil disappeared, and all the chemical shift values of the correlations were those for a typical unstructured peptide (Figure 5). In addition, all the heteronuclear nOe effects became very negative, indicating that reduced CP12 was much more mobile than the oxidized form. Thus, CP12 became completely unstructured upon reduction, in agreement with the $\mathrm{CD}$ experiments.

\section{DISCUSSION}

CP12 Is a Conserved Protein Involved in Complex Assembly. We have cloned the full sequence of CP12 cDNA 
from the unicellular green alga $C$. reinhardtii. It is highly homologous to previously sequenced CP12s from higher plants and the cyanobacterium Synechocystis PCC6803 (46) (Figure 1). All CP12s cloned to date have four cysteine residues which form two intramolecular disulfide bridges, one between cysteines 1 and 2 and the other between cysteines 3 and 4 (6). We confirmed the presence of these residues in $C$. reinhardtii $\mathrm{CP} 12$, in particular cysteines 1 and 4 , which had not been observed in the partial sequence previously obtained (5). Sequence alignment shows major differences between higher plant CP12s, cyanobacterial hypothetical and C. reinhardtii sequences. First, the presence of a proline residue between each pair of cysteines in higher plant $\mathrm{CP} 12 \mathrm{~s}$ is probably responsible for the formation of two loops in oxidized CP12 $(4,6)$. The prokaryotic and algal CP12s lack the proline residue between cysteines 1 and 2, although the proline residue between Cys 3 and 4 is present in the CP12 of all organisms. Second, the CP12s of higher plants have a set of compensating charged residues at their $\mathrm{N}$-terminus that may enable the protein to form dimers by electrostatic interactions (6). The hypothetical sequences of cyanobacteria lack the charged residues responsible for the dimerization of CP12. This "motif" may be present in $C$. reinhardtii $\mathrm{CP} 12$, but the amino acid residues putatively involved are different from those of higher plants. The most conserved part is a region of 10 residues between cysteines 2 and 3 (Figure 1), suggesting that this region may play another, yet undefined, functional role. This study is currently under investigation.

We investigated the role of CP12 in the assembly pathway of the PRK/CP12/GAPDH complex. Our in vitro reconstitution assays show that oxidized CP12, in addition to modifying GAPDH kinetic properties and probably conformation (13), also acts as a linker in the assembly of the PRK/CP12/ GAPDH complex. The formation of this complex may be a prerequisite for light-activation of the enzymes and subsequent docking of the other enzymes of the Benson-Calvin cycle as higher supramolecular complexes have been described (23-26). Complex reconstitutions with reduced CP12 were possible in the presence of $5 \mathrm{mM}$ Cys, but not $1 \mathrm{mM}$ DTT (data not shown). In the presence of the weak reducing agent ( $5 \mathrm{mM}$ Cys), the disulfide bridges form again prior to complex reconstitution. These results corroborate those obtained by Wedel et al, who showed that CP12 mutants in which Cys 1 and/or 4 were mutated into Ser failed to reconstitute the complex (6). They confirm that the presence of disulfide bridges is essential for complex reconstitution. Furthermore, alkylation of the native complex shows that $\mathrm{CP} 12$ within this complex is present under an oxidized form (data not shown).

We have performed SPR binding experiments to gain further insight into the action of CP12 and into the assembly pathway of the PRK/CP12/GAPDH complex. We have tested different combinations using two partners, one ligand and one analyte, to determine which couple of partners had the lowest dissociation constant or the highest affinity. When CP12 was used, these studies also enabled us to determine the role of CP12 in the assembly of the complex. On the basis of the following data from SPR binding experiments, in vitro reconstitution assays and the result of purifying native enzymes, we propose a model for the assembly of the PRK/ CP12/GAPDH complex and the role of CP12:

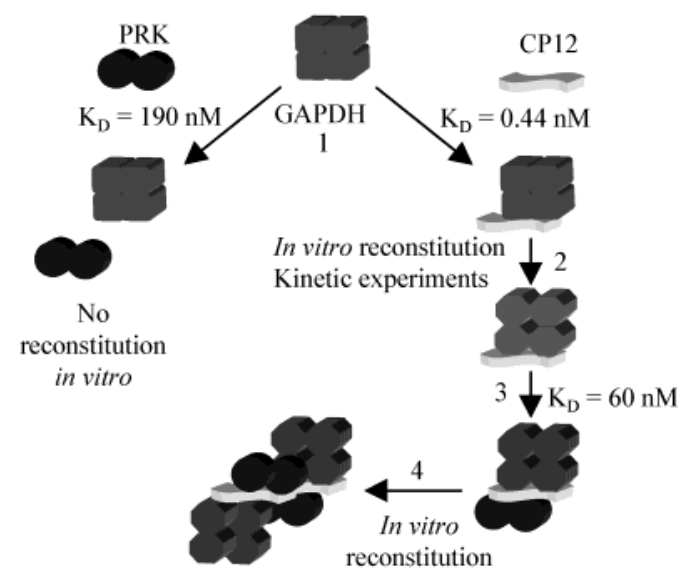

FIGURE 6: Model describing the assembly of PRK/CP12/GAPDH complex of $C$. reinhardtii. (1) GAPDH has a much higher affinity for CP12 than for PRK and thus associates with the small protein to form a very stable complex. (2) Binding of CP12 to GAPDH causes the conformation of GAPDH to change (13). According to Wedel et al (6), CP12 binds to GAPDH through its C-terminus. (3) GAPDH acquires a higher affinity for PRK and forms a subcomplex composed of one dimer of PRK, one tetramer of GADPH and one monomer of CP12. As described by Wedel et al (6), we assume that PRK interacts with the GAPDH/CP12 complex via the $\mathrm{N}$-terminus of CP12. (4) The final complex may finally be obtained by dimerization. The interactions involve CP12/CP12 contacts and additional enzyme/enzyme interactions.

(i) CP12 does not co-purify with PRK when the native enzyme is isolated. The notion of a weak interaction between PRK and CP12 is also supported by the high value of the $K_{\mathrm{d}}$ for the interaction of PRK with oxidized CP12 $(1.3 \mu \mathrm{M})$. Thus, PRK does not seem to interact with CP12 to form a stable PRK/CP12 complex.

(ii) On the contrary, native isolated GAPDH co-purifies with CP12 and forms a very stable GAPDH/CP12 complex. We have previously shown that the association of CP12 with recombinant GAPDH changes the kinetic properties of the enzyme (13). The $K_{\mathrm{d}}$ for the interaction of oxidized CP12 and GAPDH is extremely low $(0.44 \mathrm{nM})$. Furthermore, in vitro reconstitution assays using recombinant GAPDH and PRK, but no CP12, show that these two enzymes do not form a stable complex. The $K_{\mathrm{d}}$ for the interaction of recombinant GAPDH (devoid of CP12) with PRK is very high $(0.19 \mu \mathrm{M})$ compared to the one obtained for the interaction of the GAPDH/CP12 (native GAPDH) complex with PRK $(62 \mathrm{nM})$. These results show that the GAPDH that is bound to $\mathrm{CP} 12$ and has undergone a conformation change has more affinity for PRK than does GAPDH alone.

Thus, the first step in the assembly of the complex is the association of CP12 with GAPDH and the subsequent change of the conformation of the enzyme. This GAPDH/CP12 complex then becomes associated with PRK to form a halfcomplex (Figure 6), defined as one unit. This unit must then dimerize to give the native complex composed of two dimers of PRK, two tetramers of GAPDH and, probably, two monomers of CP12. The stoichiometry was determined by immunoblots (data not shown). Wedel et al proposed that dimerization involves electrostatic interactions between negatively and positively charged residues of the $\mathrm{N}$-terminal of CP12 (6). The $K_{\mathrm{d}}$ for the dimerization of oxidized CP12 by SPR is high $(51 \mu \mathrm{M})$. Yet, CP12 probably undergoes an poorly structured to structured transition upon association with GAPDH and PRK and the $K_{\mathrm{d}}$ for the dimerization of 
CP12 could change. However, prokayotic CP12s do not have such oppositely charged amino acid residues, and given the small size of $\mathrm{CP} 12$ we propose that the dimerization of the complex involves both contacts between the two enzymes and the two CP12s of each unit (Figure 6).

Redox States and Structural Transitions of CP12. We have shown that reduced $\mathrm{CP} 12$ was not able to reconstitute the complex. To link these results with a possible structural transition of CP12 upon reduction, we have analyzed the conformations of CP12 in solution by CD and NMR. The $\mathrm{CD}$ spectra show that oxidized $\mathrm{CP} 12$ has a secondary structure, mainly composed of $\alpha$ helices plus some random coils. The NMR experiments, however, indicate that CP12 does not adopt a rigid 3D structure in solution. The heteronuclear nOe experiments on His-CP12 indicate that the secondary structures are mobile one respect to another in solution. Thus, even if CP12 has secondary structures, their relative mobility makes the resolution of its 3D structure most unlikely and the NMR datasets obtained preclude the construction of a model. Since recombinant CP12 had two disulfide bridges, we reduced the protein with DTT and studied the effect of reduction on the structure of CP12. The structure of His-CP12 changes dramatically upon reduction; it loses almost all its $\alpha$-helices and becomes highly disorganized, almost unfolded and much more flexible and mobile than oxidized CP12. These results show that flexible oxidized CP12 may act as a linker in complex formation, whereas reduced and unstructured $\mathrm{CP} 12$ loses its capacity to form the complex. Such structural transitions with loss of function are also observed with small proteins, such as prion proteins. Upon transition from the normal form to the scrapie (infectious) form, the prion protein undergoes a structural transition from $\alpha$-helical to $\beta$-sheet structures, as shown by $\mathrm{CD}$ and FTIR experiments (27).

The lack of overall organization and the great flexibility of oxidized CP12 observed by NMR and CD are in good agreement with the role of this protein as linker. The flexibility and the net negative charges of CP12 may increase its reactive area and its "stickiness", compared to rigid partners. It thus facilitates specific interactions and increases the rates of specific macromolecular interactions $(28,29)$. Such behavior resembles that of "intrinsically unstructured proteins" or "IUPs" $(30-32)$. These proteins are often involved in protein - protein interactions and play major roles in cellular functions, such as DNA and RNA binding, transcriptional regulation, signal transduction, and cell cycle regulation (30). They are found in a wide variety of macromolecular complexes: Nup2p and the nuclear pore complex (33), Securin and cyclin B (34), complexins and SNARE complexes (35-37), IF1 and the regulation of ATPases (38-40), p21 or p27kip1 and regulation of CDK2, cyclin A or PCNA $(41,42)$. Like CP12, their flexibility favors the binding of several partners and the possibility of presenting larger interaction surfaces, compared to rigid partners. A recent survey of several genomes indicated that $34 \%$ of all proteins have at least one disordered segment (32). CP12 proteins thus appear as one of the few known IUPs specific to plants, in addition to Dsp16, a dehydrinrelated protein, involved in the response to abiotic stresses such as drought, low temperature, and salinity (43), and a manganese-stabilizing protein (MSP) of $33 \mathrm{kDa}$ which is part of photosystem II (44).
Interestingly, IUPs display residual secondary structures and hence are not true random-coil proteins (29). It has been speculated that these residual structures would be the same as those observed in the complex (31). CP12 probably has the same behavior and should become structured upon association with the other proteins of the complex. NMR techniques are now accurate enough to study the structure of a small $\left[{ }^{15} \mathrm{~N}\right]$ labeled protein that forms part of a macromolecular complex (45). This matter is currently under investigation.

\section{ACKNOWLEDGMENT}

We thank Renata Santos for helpful discussions and Masayuki Takahashi for advice concerning CD experiments. We also thank Jean-Jacques Montagne for MALDI-TOF mass spectrometry analyzes and Jacques d'Alayer for sequencing the N-terminus of CP12.

\section{REFERENCES}

1. Neubauer, G., King, A., Rappsilber, J., Calvio, C., Watson, M., Ajuh, P., Sleeman, J., Lamond, A., and Mann, M. (1998) Nat. Genet. 20, 46-50.

2. Rappsilber, J., Ryder, U., Lamond, A. I., and Mann, M. (2002) Genome Res. 12, 1231-1245.

3. Rout, M. P., Aitchison, J. D., Suprapto, A., Hjertaas, K., Zhao, Y., and Chait, B. T. (2000) J. Cell Biol. 148, 635-651.

4. Pohlmeyer, K., Paap, B. K., Soll, J., and Wedel, N. (1996) Plant Mol. Biol. 32, 969-978.

5. Wedel, N., and Soll, J. (1998) Proc. Natl. Acad. Sci. U.S.A. 95, 9699-9704.

6. Wedel, N., Soll, J., and Paap, B. K. (1997) Proc. Natl. Acad. Sci. U.S.A. 94, 10479-10484.

7. Nakamura, Y., Kaneko, T., Sato, S., Ikeuchi, M., Katoh, H., Sasamoto, S., Watanabe, A., Iriguchi, M., Kawashima, K., Kimura, T., Kishida, Y., Kiyokawa, C., Kohara, M., Matsumoto, M., Matsuno, A., Nakazaki, N., Shimpo, S., Sugimoto, M., Takeuchi, C., Yamada, M., and Tabata, S. (2002) DNA Res. 9, 123-130.

8. Kaneko, T., Nakamura, Y., Wolk, C. P., Kuritz, T., Sasamoto, S., Watanabe, A., Iriguchi, M., Ishikawa, A., Kawashima, K., Kimura, T., Kishida, Y., Kohara, M., Matsumoto, M., Matsuno, A., Muraki, A., Nakazaki, N., Shimpo, S., Sugimoto, M., Takazawa, M., Yamada, M., Yasuda, M., and Tabata, S. (2001) DNA Res. 8, $205-213 ; 227-253$.

9. Baalmann, E., Scheibe, R., Cerff, R., and Martin, W. (1996) Plant Mol. Biol. 32, 505-513.

10. Scheibe, R., Baalmann, E., Backhausen, J. E., Rak, C., and Vetter, S. (1996) Biochim. Biophys. Acta 1296, 228-234.

11. Avilan, L., Gontero, B., Lebreton, S., and Ricard, J. (1997) Eur. J. Biochem. 246, 78-84.

12. Mouche, F., Gontero, B., Callebaut, I., Mornon, J. P., and Boisset, N. (2002) J. Biol. Chem. 277, 6743-6749.

13. Graciet, E., Lebreton, S., Camadro, J. M., and Gontero, B. (2003) Eur. J. Biochem. 270, 129-136.

14. Graciet, E., Lebreton, S., Camadro, J. M., and Gontero, B. (2002) J. Biol. Chem. 277, 12697-12702.

15. Avilan, L., Lebreton, S., and Gontero, B. (2000) J. Biol. Chem. 275, 9447-9451.

16. Harris, E. H. (1989) The Chlamydomonas Sourcebook. A Comprehensive Guide to Biology and Laboratory Use, 1st ed.; Academic Press: San Diego.

17. Asamizu, E., Miura, K., Kucho, K., Inoue, Y., Fukuzawa, H., Ohyama, K., Nakamura, Y., and Tabata, S. (2000) DNA Res. 7, 305-307.

18. Gray, W. R. (1993) Protein Sci. 2, 1732-1748.

19. Bodenhausen, G., and Ruben, D. J. (1980) Chem. Phys. Lett. 69 , $185-189$.

20. Farrow, N. A., Muhandiram, R., Singer, A. U., Pascal, S. M., Kay, C. M., Gish, G., Shoelson, S. E., Pawson, S., Forman-Kay, J. D., and Kay, L. E. (1994) Biochemistry 33, 5984-6003.

21. Wishart, D. S., Bigam, C. G., Yao, J., F., A., Dyson, H. J., Oldfield, E., Markley, J. L., and Sykes, B. D. (1995) J. Biomol. NMR 6, $135-140$ 
22. Lebreton, S., Graciet, E., and Gontero, B. (2003) J. Biol. Chem. 278, 12078-12084.

23. Gontero, B., Cardenas, M. L., and Ricard, J. (1988) Eur. J. Biochem. 173, 437-443.

24. Anderson, L. E., Goldhaber-Gordon, I. M., Li, D., Tang, X. Y., Xiang, M., and Prakash, N. (1995) Planta 196, 245-255.

25. Süss, K. H., Arkona, C., Manteuffel, R., and Adler, K. (1993) Proc. Natl. Acad. Sci. U.SA. 90, 5514-5518.

26. Süss, K. H., Prokhorenko, I., and Adler, K. (1995) Plant Physiol. 107, 1387-1397.

27. Pan, K. M., Baldwin, M., Nguyen, J., Gasset, M., Serban, A., Groth, D., Mehlhorn, I., Huang, Z., Fletterick, R. J., Cohen, F. E., and Prusiner, S. B. (1993) Proc. Natl. Acad. Sci. U.S.A. 90, 10962-10966.

28. Pontius, B. W. (1993) Trends Biochem. Sci. 18, 181-186.

29. Uversky, V. N. (2002) Protein Sci. 11, 739-756.

30. Wright, P. E., and Dyson, H. J. (1999) J. Mol. Biol. 293, 321331.

31. Tompa, P. (2002) Trends Biochem. Sci. 27, 527-533.

32. Dyson, H. J., and Wright, P. E. (2002) Curr. Opin. Struct. Biol. $12,54-60$.

33. Denning, D. P., Uversky, V., Patel, S. S., Fink, A. L., and Rexach, M. (2002) J. Biol. Chem. 277, 33447-33455.

34. Cox, C. J., Dutta, K., Petri, E. T., Hwang, W. C., Lin, Y., Pascal, S. M., and Basavappa, R. (2002) FEBS Lett. 527, 303-308.
35. Pabst, S., Hazzard, J. W., Antonin, W., Sudhof, T. C., Jahn, R., Rizo, J., and Fasshauer, D. (2000) J. Biol. Chem. 275, 1980819818.

36. Pabst, S., Margittai, M., Vainius, D., Langen, R., Jahn, R., and Fasshauer, D. (2002) J. Biol. Chem. 277, 7838-7848.

37. Jahn, R., and Sudhof, T. C. (1999) Аnпи. Rev. Biochem. 68, 863911.

38. Solaini, G., Baracca, A., Gabellieri, E., and Lenaz, G. (1997) Biochem. J. 327, 443-448.

39. Cabezon, E., Runswick, M. J., W., L. A. G., and Walker, J. E. (2001) EMBO J. 20, 6990-6996.

40. Cabezon, E., Arechaga, I., Butler, P. J. G., and Walker, J. E. (2000) J. Biol. Chem. 275, 28353-28355.

41. Russo, A. A., Jeffrey, P. D., Patten, A. K., Massague, J., and Pavlevitch, N. P. (1996) Nature 382, 325-331.

42. Gulbis, J. M., Kelman, Z., Hurwitz, J., M., O. D., and Kuriyan, J. (1996) Cell 87, 297-306

43. Lisse, T., Bartels, D., Kalbitzer, H. R., and Jaenicke, R. (1996) Biol. Chem. 377, 555-561.

44. Lykadis-Simantiris, N., Hutchison, R. S., Betts, S. D., Barry, B. A., and Yocum, C. F. (1999) Biochemistry 38, 404-414.

45. Fiaux, J., Bertelsen, E. B., Horwich, A. L., and Wüthrich, K. (2002) Nature 418, 207-211.

BI034474X 